\title{
Weak Bisimulations for Coalgebras over Ordered Functors
}

\author{
Tomasz Brengos ${ }^{\star}$ \\ Faculty of Mathematics and Information Sciences \\ Warsaw University of Technology \\ Koszykowa 75 \\ 00-662 Warszawa, Poland \\ t.brengos@mini.pw.edu.pl
}

\begin{abstract}
The aim of this paper is to introduce a coalgebraic setting in which it is possible to generalize and compare the two known approaches to defining weak bisimulation for labelled transition systems. We introduce two definitions of weak bisimulation for coalgebras over ordered functors, show their properties and give sufficient conditions for them to coincide. We formulate a weak coinduction principle.
\end{abstract}

Keywords: coalgebra, bisimulation, saturator, weak bisimulation, weak coinduction.

\section{Introduction}

The notion of a strong bisimulation for different transition systems plays an important role in theoretical computer science. A weak bisimulation is a relaxation of this notion by allowing silent, unobservable transitions. It is a well established notion for many deterministic and probabilistic transition systems (see [1], 4], 7], 8]). For many state-based systems one can equivalently introduce weak bisimulation in two different ways one of which has computational advantages over the other. To be more precise we will demonstrate this phenomenon on labelled transition systems. By a labelled transition system (or LTS in short) we mean a tuple $\langle A, \Sigma, \rightarrow\rangle$, where $A$ is a set of states, $\Sigma$ is a non-empty set called an alphabet and $\rightarrow$ is a subset of $A \times \Sigma \times A$ and is called a transition. For an LTS $\langle A, \Sigma, \rightarrow\rangle$ and $s \in \Sigma$ we define a relation on $A$ by

$$
\stackrel{s}{\rightarrow}:=\left\{\left(a, a^{\prime}\right) \in A^{2} \mid\left(a, s, a^{\prime}\right) \in \rightarrow\right\} .
$$

For a fixed alphabet letter $\tau \in \Sigma$, representing a silent, unobservable transition label, and an LTS $\langle A, \Sigma, \rightarrow\rangle$ let $\stackrel{\tau^{*}}{\rightarrow}$ be the reflexive and transitive closure of the relation $\stackrel{\tau}{\rightarrow}$. The following definition of a weak bisimulation for LTS can be found in [4.

\footnotetext{
* This work has been supported by the European Union in the framework of European Social Fund through the Warsaw University of Technology Development Programme and the grant of Warsaw University of Technology no. 504M for young researchers.
} 
Definition 1. A relation $R \subseteq A \times A$ is a weak bisimulation if it satisfies the following conditions. For $(a, b) \in R$ and $\sigma \neq \tau$ if $a \stackrel{\tau^{*}}{\rightarrow} \circ \stackrel{\sigma}{\rightarrow} \circ \stackrel{\tau^{*}}{\rightarrow} a^{\prime}$ then there is $b^{\prime} \in A$ such that $b \stackrel{\tau^{*}}{\rightarrow} \circ \stackrel{\sigma}{\rightarrow} \circ \stackrel{\tau^{*}}{\rightarrow} b^{\prime}$ with $\left(a^{\prime}, b^{\prime}\right) \in R$ and conversely, for $b \stackrel{\tau^{*}}{\rightarrow} \circ \stackrel{\sigma}{\rightarrow} \circ \stackrel{\tau^{*}}{\rightarrow} b^{\prime \prime}$ there is $a^{\prime \prime} \in A$ such that $a \stackrel{\tau^{*}}{\rightarrow} \circ \stackrel{\sigma}{\rightarrow} \circ \stackrel{\tau^{*}}{\rightarrow} a^{\prime \prime}$ and $\left(a^{\prime \prime}, b^{\prime \prime}\right) \in R$. Moreover, for $\sigma=\tau$ if $a \stackrel{\tau^{*}}{\rightarrow} a^{\prime}$ then $b \stackrel{\tau^{*}}{\rightarrow} b^{\prime}$ for some $b^{\prime} \in B$ with $\left(a^{\prime}, b^{\prime}\right) \in R$ and conversely, if $b \stackrel{\tau}{\rightarrow} b^{\prime \prime}$ then $a \stackrel{\tau^{*}}{\rightarrow} a^{\prime \prime}$ for some $a^{\prime \prime} \in A$ and $\left(a^{\prime \prime}, b^{\prime \prime}\right) \in R$.

It is easily shown we can equivalently restate the definition of a weak bisimulation as follows.

Definition 2. A relation $R \subseteq A \times A$ is a weak bisimulation if it satisfies the following condition. If $(a, b) \in R$ then for $\sigma \neq \tau$ if $a \stackrel{\sigma}{\rightarrow} a^{\prime}$ then $b \stackrel{\tau}{\rightarrow} \stackrel{*}{*} \stackrel{\sigma}{\rightarrow} \circ \stackrel{\tau}{\rightarrow}{ }^{*} b^{\prime}$ for some $b^{\prime} \in A$ and $\left(a^{\prime}, b^{\prime}\right) \in R$, for $\sigma=\tau$ if $a \stackrel{\tau}{\rightarrow} a^{\prime}$ then $b \stackrel{\tau}{\rightarrow}{ }^{*} b^{\prime}$ for some $b^{\prime} \in A$ and $\left(a^{\prime}, b^{\prime}\right) \in R$, moreover for $\sigma \neq \tau$ if $b \stackrel{\sigma}{\rightarrow} b^{\prime}$ then $a \stackrel{\tau}{\rightarrow}{ }^{*} \circ \stackrel{\sigma}{\rightarrow} \circ \stackrel{\tau}{\rightarrow}^{*} a^{\prime}$ for some $a^{\prime} \in A$ and $\left(a^{\prime}, b^{\prime}\right) \in R$, for $\sigma=\tau$ if $b \stackrel{\tau}{\rightarrow} b^{\prime}$ then $a \stackrel{\tau}{\rightarrow}^{*} a^{\prime}$ for some $a^{\prime} \in A$ and $\left(a^{\prime}, b^{\prime}\right) \in R$.

From the point of view of computation and automatic reasoning the latter approach to defining weak bisimulation is better since, unlike the former, it does not require the knowledge of the full saturated transition. Indeed, in order to show that two states $a, b \in A$ of a labelled transition system $\langle A, \Sigma, \rightarrow\rangle$ are weakly bisimilar in the sense of Definition 10 one needs to consider all states $a^{\prime} \in A$ reachable from $a$ via the saturated transitions $\stackrel{\tau}{\rightarrow} \stackrel{*}{\circ} \stackrel{\sigma}{\rightarrow} \circ \stackrel{\tau}{\rightarrow}$ or $\stackrel{\tau}{\rightarrow}$ and compare them with similar states reachable from $b$. Whereas, to prove that two states $a, b \in A$ are weakly bisimilar in the sense of Definition 2 one needs to consider all states reachable from $a$ via single step transitions $\stackrel{\sigma}{\rightarrow}$ and compare them with some states reachable from $b$ via the saturated transitions.

The notion of a strong bisimulation, unlike the weak bisimulation, has been well captured coalgebraically (see e.g. [2, [11]). Different approaches to defining weak bisimulations for coalgebras have been presented. The earliest paper is [10, where the author studies weak bisimulations for while programs. In [5] the author introduces a definition of weak bisimulation for coalgebras by translating a coalgebraic structure into an LTS. This construction works for coalgebras over a large class of functors but does not cover the distribution functor, hence it is not applicable to different types of probabilistic systems. In [6], weak bisimulations are introduced via weak homomorphisms. As noted in 9 this construction does not lead to intuitive results for probabilistic systems. Finally, in 9 the authors present a definition of weak bisimulation for classes of coalgebras over functors obtained from bifunctors. Here, weak bisimulation of a system is defined as a strong bisimulation of a transformed system. First of all, it is worth noting that, although very interesting, neither of the approaches cited above expresses coalgebraically the computational advantages of Definition 2 over Definition 1. Secondly, all of them require to explicitly work with observable and unobservable part of the coalgebraic structure. The method of defining weak bisimulation presented in this paper only requires that a saturator is given and no explicit knowledge of silent and visible part of computation is neccessary. 
The aim of this paper is to introduce a coalgebraic setting in which we can define weak bisimulation in two ways generalizing Definition 1 and Definition 2 and compare them. Additionally, we formulate a weak coinduction principle. The paper is organized as follows. In Section 2 we present basic definitions and properties from known universal coalgebra. In Section 3 we present a definition of a saturator and present some natural and well-known examples of saturators. In Section 4 we give two approaches to defining weak bisimulation via saturators and show their properties. Finally, Section 5 is devoted to formulation of a weak coinduction rule.

\section{Basic Notions and Properties}

Let Set be the category of all sets and mappings between them. Let $F$ : Set $\rightarrow$ Set be a functor. An $F$-coalgebra is a tuple $\langle A, \alpha\rangle$, where $A$ is a set and $\alpha$ is a mapping $\alpha: A \rightarrow F A$. The set $A$ is called a carrier and the mapping $\alpha$ is called a structure of the coalgebra $\langle A, \alpha\rangle$.

A homomorphism from an $F$-coalgebra $\langle A, \alpha\rangle$ to a $F$-coalgebra $\langle B, \beta\rangle$ is a mapping $f: A \rightarrow B$ such that $T(f) \circ \alpha=\beta \circ f$.

An $F$-coalgebra $\langle S, \sigma\rangle$ is said to be a subcoalgebra of an $F$-coalgebra $\langle A, \alpha\rangle$ whenever there is an injective homomorphism from $\langle S, \sigma\rangle$ into $\langle A, \alpha\rangle$. This fact is denoted by $\langle S, \sigma\rangle \leq\langle A, \alpha\rangle$.

We denote the disjoint union of a family $\left\{X_{j}\right\}_{j \in J}$ of sets by $\Sigma_{j \in J} X_{j}$. Let $\left\{\left\langle A_{i}, \alpha_{i}\right\rangle\right\}_{i \in I}$ be a family of $F$-coalgebras. The disjoint sum $\Sigma_{i \in I}\left\langle A_{i}, \alpha_{i}\right\rangle$ of the family $\left\{\left\langle A_{i}, \alpha_{i}\right\rangle\right\}_{i \in I}$ of $F$-coalgebras is an $F$-coalgebra defined as follows. The carrier set of the disjoint sum $\Sigma_{i \in I}\left\langle A_{i}, \alpha_{i}\right\rangle$ is the disjoint union of the carriers of $\left\langle A_{i}, \alpha_{i}\right\rangle$, i.e. $A:=\Sigma_{i \in I} A_{i}$. The structure $\alpha$ of the disjoint sum $\Sigma_{i \in I}\left\langle A_{i}, \alpha_{i}\right\rangle$ is defined as

$$
\alpha: A \rightarrow F A ; A_{i} \ni a \mapsto F\left(e_{i}\right) \circ \alpha_{i}(a),
$$

where $e_{i}: A_{i} \rightarrow A ; a \mapsto(a, i)$ for any $i \in I$.

A functor $F$ : Set $\rightarrow$ Set preserves pullbacks if for any mappings $f: A \rightarrow B$ and $g: C \rightarrow B$ and their pullback $P(f, g)=\{(a, c) \in A \times C \mid f(a)=g(c)\}$ with $\pi_{1}$ and $\pi_{2}$ the following diagram is a pullback diagram.

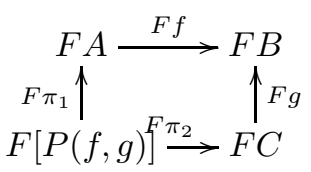

We say that $F$ weakly preserves pullbacks if the diagram above is a weak pullback. A functor $F$ (weakly) preserves kernel pairs if it (weakly) preserves pullbacks $P(f, f), \pi_{1}, \pi_{2}$ for any mapping $f: A \rightarrow B$. For a detailed analysis of pullback and kernel pair preservations the reader is referred to [2].

Let Pos be the category of all posets and monotonic mappings. Note that there is a forgetful functor $U:$ Pos $\rightarrow$ Set assigning to each poset $(X, \leq)$ the underlying set $X$ and to each monotonic map $f:(X, \leq) \rightarrow(Y, \leq)$ the map $f: X \rightarrow Y$. 
From now on we assume that a functor $F$ we work with is $F$ : Set $\rightarrow$ Pos. We may naturally assign to $F$ its composition $\bar{F}=U \circ F$ with the forgetful functor $U:$ Pos $\rightarrow$ Set. For the sake of simplicity of notation most of the times we will identify the functor $F$ : Set $\rightarrow$ Pos with the Set-endofunctor $\bar{F}=U \circ F$ and write $F$ to denote both $F$ and $\bar{F}$. Considering set-based coalgebras over functors whose codomain category is a concrete category different from Set is not a new approach. A similar one has been adopted by e.g. J. Hughes and B. Jacobs in [3] when definining simulations for coalgebras.

Example 1. The powerset endofunctor $\mathcal{P}:$ Set $\rightarrow$ Set can be considered a functor $\mathcal{P}:$ Set $\rightarrow$ Pos which assigns to any set $X$ the poset $(\mathcal{P}(X), \subseteq)$ and to any map $f: X \rightarrow Y$ the order preserving map $\mathcal{P}(f)$.

Example 2. For any functor $H:$ Set $\rightarrow$ Set the composition $\mathcal{P} H$ may be regarded as a functor $\mathcal{P} H$ : Set $\rightarrow$ Pos with a natural ordering given by inclusion. In this paper we will focus our attention on coalgebras over the following functors:

$-\mathcal{P}(\Sigma \times \mathcal{I} d)$

$-\mathcal{P}(\Sigma+\mathcal{I} d)$

$-\mathcal{P}(\Sigma \times \mathcal{D})$

where $\mathcal{D}$ is the distribution functor, i.e. a functor which assigns to any set $X$ the set $\mathcal{D} X:=\left\{\mu: X \rightarrow[0,1] \mid \sum_{x \in X} \mu(x)=1\right\}$ of discrete measures and to any mapping $f: X \rightarrow Y$ a mapping $\mathcal{D} f: \mathcal{D} X \rightarrow \mathcal{D} Y$, which assigns to any measure $\mu \in \mathcal{D} X$ the measure $\mathcal{D} f(\mu): Y \rightarrow[0,1]$ such that

$$
\mathcal{D} f(\mu)(y)=\sum_{f(x)=y} \mu(x) \text { for any } y \in Y \text {. }
$$

The coalgebras over the first functor are exactly labelled transition systems. The coalgebras for $\mathcal{P}(\Sigma+\mathcal{I} d)$ expand a class of coalgebras studied by J. Rutten in [10. Finally, the $\mathcal{P}(\Sigma \times \mathcal{D})$-coalgebras generalize the class of simple Segala systems introduced and thoroughly studied in [7, 8].

For a functor $F$ : Set $\rightarrow$ Pos and for any sets $X, Y$ we introduce an order on the set $\operatorname{Hom}(X, F Y)$ as follows. For $f, g \in \operatorname{Hom}(X, F Y)$ put

$$
f \leq g \stackrel{\text { def }}{\Longleftrightarrow} f(x) \leq_{F Y} g(x) \text { for any } x \in X .
$$

Given $f: X \rightarrow Y, \alpha: X \rightarrow F Z, g: Z \rightarrow U$ and $\beta: Y \rightarrow F U$ an inequality $F g \circ \alpha \leq \beta \circ f$ will be denoted by a diagram on the left and an equality $F g \circ \alpha=$ $\beta \circ f$ will be denoted by a diagram on the right:

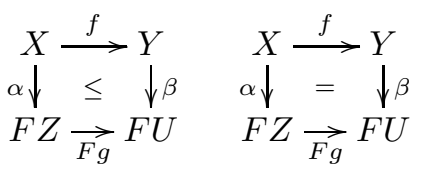


Lemma 1. Let $\alpha, \beta \in H o m(X, F Y)$ and let $f: Z \rightarrow X$ be an epimorphism in Set. If $\alpha \circ f \leq \beta \circ f$ in $\operatorname{Hom}(Z, F Y)$ then $\alpha \leq \beta$ in $\operatorname{Hom}(X, F Y)$.

Proof. Since $\alpha \circ f \leq \beta \circ f$ then for any $z \in Z$ we have $\alpha(f(z)) \leq_{F Y} \beta(f(z))$. Because $f(Z)=X$ we have $\alpha(x) \leq_{F Y} \beta(x)$ for any $x \in X$. Hence, $\alpha \leq \beta$ in $\operatorname{Hom}(X, F Y)$.

\section{Coalgebraic Operators and Saturators}

Definition 3. Let $U: \operatorname{Set}_{F} \rightarrow$ Set be the forgetful functor and let $\mathrm{C}$ be full subcategory of the category of $F$-coalgebras and homomorphisms between them which is closed under taking inverse images of homomorphisms, i.e. if $\langle B, \beta\rangle \in$ $\mathrm{C}$ and there is a homomorphism $f:\langle A, \alpha\rangle \rightarrow\langle B, \beta\rangle$ for $\langle A, \alpha\rangle \in \operatorname{Set}_{F}$ then $\langle A, \alpha\rangle \in \mathrm{C}$. A coalgebraic operator $\mathfrak{o}$ with respect to a class $\mathrm{C}$ is a functor $\mathfrak{o}: \mathrm{C} \rightarrow \operatorname{Set}_{F}$ such that the following diagram commutes:

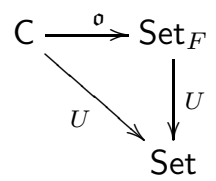

In other words, if $f: A \rightarrow B$ is a homomorphism between two $F$-coalgebras $\langle A, \alpha\rangle$ and $\langle B, \beta\rangle$ belonging to $\mathrm{C}$ then $f$ is a homomorphism between $\langle A, \mathfrak{o} \alpha\rangle$ and $\langle B, \mathfrak{o} \beta\rangle$, i.e.

$$
\begin{gathered}
A \stackrel{f}{\longrightarrow} B \\
\alpha \downarrow=\downarrow \beta \\
F B \underset{F f}{\rightarrow} F B
\end{gathered} \quad \begin{gathered}
A \stackrel{f}{\rightarrow} B= \\
F A \underset{F f}{\longrightarrow} F B
\end{gathered}
$$

We say that a coalgebraic operator $\mathfrak{s}$ with respect to a class $\mathrm{C}$ is a saturator if for any two $F$-coalgebras $\langle A, \alpha\rangle,\langle B, \beta\rangle$ from $\mathrm{C}$ and any mapping $f: A \rightarrow B$ the inequality $F f \circ \alpha \leq \mathfrak{s} \beta \circ f$ is equivalent to $F f \circ \mathfrak{s} \alpha \leq \mathfrak{s} \beta \circ f$. We may express the property in diagrams as follows:

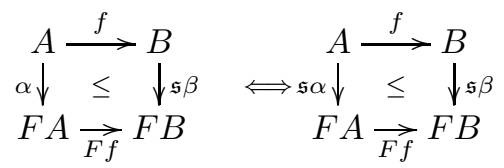

Lemma 2. Let $\mathfrak{s}: \mathrm{C} \rightarrow \operatorname{Set}_{F}$ be an operator w.r.t. a full subcategory $\mathrm{C}$ of Set $_{F}$ and additionally let $\mathfrak{s}(\mathrm{C}) \subseteq \mathrm{C}$. Then $\mathfrak{s}$ is a saturator if and only if it satisfies the following three properties:

$-\alpha \leq \mathfrak{s} \alpha$ for any coalgebra $\langle A, \alpha\rangle \in \mathrm{C}$ (extensivity),

$-\mathfrak{s} \circ \mathfrak{s}=\mathfrak{s}$ (idempotency),

- if $F f \circ \alpha \leq \beta \circ f$ then $F f \circ \mathfrak{s} \alpha \leq \mathfrak{s} \beta \circ f$ for any $f: X \rightarrow Y$ (monotonicity): 


$$
\begin{gathered}
A \stackrel{f}{\longrightarrow} B \quad A \stackrel{f}{\longrightarrow} B \\
\alpha \downarrow \leq \downarrow \beta \\
F A \underset{F f}{\longrightarrow} F B
\end{gathered} \quad \begin{gathered}
\text { s } \alpha \downarrow \leq \downarrow \\
F A \underset{F f}{\rightarrow} F B
\end{gathered}
$$

The intuition behind the notion of a saturator is the following. Given a coalgebraic structure $\alpha: A \rightarrow F A$ it contains some information about observable and unobservable single step transitions. The process of saturating a structure intuitively boils down to adding additional information to $\alpha$ about multiple compositions of unobservable steps and a single composition of observable transitions (see examples below). Since for any set $A$ the set $F A$ is intuitively considered as the set of all possible outcomes of a computation, the partial order $\leq$ on $F A$ compares those outcomes. In particular, the property of extensivity of a saturator means the saturated structure $\mathfrak{s} \alpha$ contains at least the same information about single-step transitions as $\alpha$ (in the sense of the partial order $\leq$ ). Idempotency means that the process of adding new information to $\alpha$ by saturating it ends after one iteration. Finally, monotonicity is self-explanatory.

Example 3. Let $\Sigma$ be a non-empty set. Any LTS $\langle A, \Sigma, \rightarrow\rangle$ may be represented as a $\mathcal{P}(\Sigma \times \mathcal{I} d)$-coalgebra $\langle A, \alpha\rangle$ as follows. We define $\alpha: A \rightarrow \mathcal{P}(\Sigma \times A)$ by:

$$
\left(\sigma, a^{\prime}\right) \in \alpha(a) \Longleftrightarrow a \stackrel{\sigma}{\rightarrow} a^{\prime}
$$

Let $\tau \in \Sigma$ be a silent transition label. For a coalgebra structure $\alpha: A \rightarrow \mathcal{P}(\Sigma \times A)$ we define its saturation $\mathfrak{s} \alpha: A \rightarrow \mathcal{P}(\Sigma \times A)$ as follows. For any element $a \in A$ put

$$
\mathfrak{s} \alpha(a):=\alpha(a) \cup\left\{\left(\tau, a^{\prime}\right) \mid a \stackrel{\tau^{*}}{\rightarrow} a^{\prime}\right\} \cup\left\{\left(\sigma, a^{\prime}\right) \mid a \stackrel{\tau^{*}}{\rightarrow} \circ \stackrel{\sigma}{\rightarrow} \circ \stackrel{\tau^{*}}{\rightarrow} a^{\prime} \text { for } \sigma \neq \tau\right\} .
$$

Verifying that $\mathfrak{s}: \operatorname{Set}_{\mathcal{P}(\Sigma \times \mathcal{I} d)} \rightarrow \operatorname{Set}_{\mathcal{P}(\Sigma \times \mathcal{I} d)} ;\langle A, \alpha\rangle \mapsto\langle A, \mathfrak{s} \alpha\rangle$ is a coalgebraic saturator with respect to the class of all $\mathcal{P}(\Sigma \times \mathcal{I} d)$-coalgebras is left to the reader.

Example 4. Consider the functor $F=\mathcal{P}(\Sigma+\mathcal{I} d)$. Let $\alpha: A \rightarrow \mathcal{P}(\Sigma+A)$ be a structure of an $F$-coalgebra $\langle A, \alpha\rangle$. For the sake of simplicity of notation for any $a \in A$ let $\eta(a):=\alpha(a) \cap A$ and $\theta(a):=\alpha(a) \cap \Sigma$. Put $\eta^{*}(a):=\{a\} \cup \bigcup_{n \in \mathbb{N}} \eta^{n}(a)$, where $\eta^{n}(a):=\eta\left(\eta^{n-1}(a)\right)$ for $n>1$ and $\theta^{*}(a):=\theta\left(\eta^{*}(a)\right)$. Define the saturation $\mathfrak{s} \alpha: A \rightarrow F A$ as follows:

$$
\mathfrak{s} \alpha(a):=\eta^{*}(a) \cup \theta^{*}(a) \text { for any } a \in A .
$$

The assignment $\mathfrak{s}$ is a coalgebraic saturator with respect to the class of all $F$ coalgebras.

Example 5. For the functor $F=\mathcal{P}(\Sigma \times \mathcal{D})$, an $F$-coalgebra $\langle A, \alpha\rangle$, a state $a \in A$ and $\sigma \in \Sigma$ we write $a \stackrel{\sigma}{\rightarrow} \mu$ if $(\sigma, \mu) \in \alpha(a)$. For a state $a \in A$ and a measure $\nu \in \mathcal{D}(\Sigma \times A)$ a pair $(a, \nu)$ is called a step in $\langle A, \alpha\rangle$ only if there is $\sigma \in A$ and $\mu \in \mathcal{D} A$ such that $a \stackrel{\sigma}{\rightarrow} \mu$ and $\nu\left(\sigma, a^{\prime}\right)=\mu\left(a^{\prime}\right)$ for any $a^{\prime} \in A$. A combined 
step in $\langle A, \alpha\rangle$ is a pair $(a, \nu)$, where $a \in A$ and $\nu \in \mathcal{D}(\Sigma \times A)$ for which there is a countable family of non-negative numbers $\left\{p_{i}\right\}_{i \in I}$ such that $\sum_{i \in I} p_{i}=1$ and a countable family of steps $\left\{\left(a, \nu_{i}\right)\right\}_{i \in I}$ in $\langle A, \alpha\rangle$ such that $\nu=\sum_{i \in I} p_{i} \cdot \nu_{i}$. The definition of a combined step is a slight modification of a similar definition presented in [7]. The notion of weak arrows $\stackrel{\sigma}{\Longrightarrow} P$ remains the same regardless of the small difference between the two definitions. Let $\tau \in \Sigma$ be the invisible transition. As in [7] for any $\sigma \in \Sigma$ we write $a \stackrel{\sigma}{\Longrightarrow} P \mu$ whenever $\sigma=\tau$ and $\mu \in \mathcal{D} A$ for which $\mu(a)=1$ or there is a combined step $(a, \nu)$ in $\langle A, \alpha\rangle$ such that if $\left(\sigma^{\prime}, a^{\prime}\right) \notin\{\sigma, \tau\} \times A$ then $\nu\left(\sigma^{\prime}, a^{\prime}\right)=0$ and $\mu=\sum_{\left(\sigma^{\prime}, a^{\prime}\right) \in\{\sigma, \tau\} \times A} \nu\left(\sigma^{\prime}, a^{\prime}\right) \cdot \mu_{\left(\sigma^{\prime}, a^{\prime}\right)}$ and if $\sigma^{\prime}=\sigma$ then $a^{\prime} \stackrel{\tau}{\Longrightarrow} P \mu_{\left(\sigma^{\prime}, a^{\prime}\right)}$ otherwise $\sigma^{\prime}=\tau$ and $a^{\prime} \stackrel{\sigma}{\Longrightarrow}{ }_{P} \mu_{\left(\sigma^{\prime}, a^{\prime}\right)}$. Now, define $\mathfrak{s} \alpha: A \rightarrow F A$ by putting $\mathfrak{s} \alpha(a):=\{(\sigma, \mu) \mid a \stackrel{\sigma}{\Longrightarrow} P \mu\}$ for any $a \in A$. A proof that $\mathfrak{s}$ is a coalgebraic saturator is left to the reader.

\section{Two Approaches to Defining Weak Bisimulation}

In this section we assume that $\langle A, \alpha\rangle$ and $\langle B, \beta\rangle$ are members of the class $\mathrm{C}$.

Definition 4. A relation $R \subseteq A \times B$ is called a weak bisimulation provided that there is a structure $\gamma_{1}: R \rightarrow F R$ and a structure $\gamma_{2}: R \rightarrow F R$ for which:

$-\alpha \circ \pi_{1}=F \pi_{1} \circ \gamma_{1}$ and $F \pi_{2} \circ \gamma_{1} \leq \mathfrak{s} \beta \circ \pi_{2}$,

$-\beta \circ \pi_{2}=F \pi_{2} \circ \gamma_{2}$ and $F \pi_{1} \circ \gamma_{2} \leq \mathfrak{s} \alpha \circ \pi_{1}$.
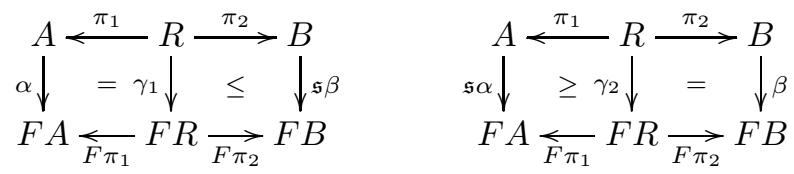

Example 6. Consider the LTS functor $F=\mathcal{P}(\Sigma \times \mathcal{I} d)$ and the saturator $\mathfrak{s}$ introduced in Example 3. Let $\langle A, \alpha\rangle$ be an $F$-coalgebra. Consider a relation $R \subseteq A \times A$ which satisfies the assumptions of Definition 4. This means that if $(a, b) \in R$ then there is $\gamma_{1}: R \rightarrow \mathcal{P}(\Sigma \times R)$ such that $\alpha(a)=F\left(\pi_{1}\right)\left(\gamma_{1}(a, b)\right)$ and $F\left(\pi_{2}\right)\left(\gamma_{1}(a, b)\right) \subseteq \mathfrak{s} \alpha(b)$. In other words, there is a subset $S \subseteq \Sigma \times R$ such that $\gamma_{1}(a, b)=S$ and $F\left(\pi_{1}\right)(S)=\alpha(a)$ and $F\left(\pi_{2}\right)(S) \subseteq \mathfrak{s} \alpha(b)$. This means that for any $\left(\sigma, a^{\prime}\right) \in \alpha(a)$ there is $b^{\prime} \in A$ such that $\left(\sigma, b^{\prime}\right) \in \mathfrak{s} \alpha(b)$ and $\left(a^{\prime}, b^{\prime}\right) \in R$. Hence, if $\sigma=\tau$ then $a \stackrel{\tau}{\rightarrow} a^{\prime}$ implies $b \stackrel{\tau^{*}}{\rightarrow} b^{\prime}$ and $\left(a^{\prime}, b^{\prime}\right) \in R$ otherwise $a \stackrel{\sigma}{\rightarrow} a^{\prime}$ implies $b \stackrel{\tau^{*} \sigma \tau^{*}}{\rightarrow} b^{\prime}$ and $\left(a^{\prime}, b^{\prime}\right) \in R$. The second condition from Definition 4 gives us the following assertion. If $(a, b) \in R$ and $b \stackrel{\tau}{\rightarrow} b^{\prime}$ then there is $a^{\prime} \in A$ such that $a \stackrel{\tau^{*}}{\rightarrow} a^{\prime}$ and $\left(a^{\prime}, b^{\prime}\right) \in R$. Moreover, if for $\sigma \neq \tau$ we have $b \stackrel{\sigma}{\rightarrow} b^{\prime}$ then there is $a^{\prime} \in A$ such that $a \stackrel{\tau^{*} \sigma \tau^{*}}{\rightarrow} a^{\prime}$ and $\left(a^{\prime}, b^{\prime}\right) \in R$. We see that this is exactly the condition presented in Definition 2 .

Example \%. Consider the functor $F=\mathcal{P}(\Sigma+\mathcal{I} d)$ and the saturator $\mathfrak{s}$ from Example 4. Since the functor $\Sigma+\mathcal{I} d$ is a subfunctor of $F$ take an $F$-coalgebra 
$\langle A, \alpha\rangle$ which is a $\Sigma+\mathcal{I} d$-coalgebra, i.e. the structure $\alpha$ is a mapping $\alpha: A \rightarrow$ $\Sigma+A$. Now take a relation $R \in A \times A$ which satisfies the assumptions presented in Definition 4 and let $(a, b) \in R$. This means that there is a structure $\gamma_{1}$ : $R \rightarrow \mathcal{P}(\Sigma+R)$ such that $F\left(\pi_{1}\right)\left(\gamma_{1}(a, b)\right)=\alpha(a)$ and $F\left(\pi_{2}\right)\left(\gamma_{1}(a, b)\right) \subseteq \mathfrak{s} \alpha(a)$. In other words there is a pair $X, S$ of subsets, where $X \subseteq R$ and $S \subseteq \Sigma$, and $F\left(\pi_{1}\right)(X \cup S)=\left(\pi_{1}(X) \cup S\right)=\alpha(a)$ and $F\left(\pi_{2}\right)(X \cup S)=\left(\pi_{2}(X) \cup S\right) \subseteq \mathfrak{s} \alpha(a)$. If $\alpha(a)=a^{\prime} \in A$ then this means that $\pi_{1}(X)=\left\{a^{\prime}\right\}$ and $S=\varnothing$. Hence, there is $b^{\prime} \in A$ such that $\left(a^{\prime}, b^{\prime}\right) \subseteq X \subseteq R$ and $b^{\prime} \in \eta^{*}(b)=\left\{b^{\prime \prime} \mid b \rightarrow^{*} b^{\prime \prime}\right\}$. If $\alpha(a)=\sigma \in \Sigma$ then $\pi_{1}(X)=\varnothing$, and hence $X=\varnothing, S=\{\sigma\}$. Therefore, $\sigma \in \theta^{*}(b)$. It follows that there is $b^{\prime} \in B$ such that $b \rightarrow^{*} b^{\prime}$ and $b^{\prime} \downarrow \sigma$. By the second condition of Definition 4 we infer that if $b \rightarrow b^{\prime}$ then there is $a^{\prime} \in A$ such that $a \rightarrow^{*} a^{\prime}$ and $\left(a^{\prime}, b^{\prime}\right) \in R$. Otherwise if $b \downarrow \sigma \in \Sigma$ then there is $a^{\prime} \in A$ such that $a \rightarrow^{*} a^{\prime}$ and $a^{\prime} \downarrow \sigma$. This definition coincides with a definition of weak bisimulation between $\Sigma+\mathcal{I} d$-coalgebras presented in [10].

Example 8. Let $F=\mathcal{P}(\Sigma \times \mathcal{D})$ and consider the saturator $\mathfrak{s}: \operatorname{Set}_{F} \rightarrow \operatorname{Set}_{F}$ defined in Example 5. It is easy to see that for two simple Segala systems $\langle A, \alpha\rangle,\langle B, \beta\rangle$ a relation $R \subseteq A \times B$ is a weak bisimulation provided that the following condition holds. If $(a, b) \in R$ and $a \stackrel{\sigma}{\rightarrow} \mu$ then $b \stackrel{\sigma}{\Longrightarrow} P \mu^{\prime}$ and $\left(\mu, \mu^{\prime}\right) \in\left(F \pi_{1}, F \pi_{2}\right)(\mathcal{D} R)$. Moreover, if $(a, b) \in R$ and $b \stackrel{\sigma}{\rightarrow} \mu^{\prime}$ then $a \stackrel{\sigma}{\Longrightarrow} P \mu$ and $\left(\mu, \mu^{\prime}\right) \in\left(F \pi_{1}, F \pi_{2}\right)(\mathcal{D} R)$. This definition coincides with the one presented in [7, 8].

Proposition 1. Let $R \subseteq A \times B$ be a standard bisimulation between $\langle A, \alpha\rangle$ and $\langle B, \beta\rangle$. Then $R$ is also a weak bisimulation between $\langle A, \alpha\rangle$ and $\langle B, \beta\rangle$.

Theorem 1. If a relation $R \subseteq A \times B$ is a weak bisimulation between $\langle A, \alpha\rangle$ and $\langle B, \beta\rangle$ then $R^{-1}=\{(b, a) \mid(a, b) \in R\}$ is a weak bisimulation between $\langle B, \beta\rangle$ and $\langle A, \alpha\rangle$.

Theorem 2. If all members of a family $\left\{R_{i}\right\}_{i \in I}$ of relations $R_{i} \subseteq A \times B$ are weak bisimulations between $\langle A, \alpha\rangle$ and $\langle B, \beta\rangle$ then $\bigcup_{i \in I} R_{i}$ is also a weak bisimulation between $\langle A, \alpha\rangle$ and $\langle B, \beta\rangle$.

Proof. Let $\left\{R_{i}\right\}_{i \in I}$ together with $\gamma_{1}^{i}: R_{i} \rightarrow F R_{i}$ and $\gamma_{2}^{i}: R_{i} \rightarrow F R_{i}$ be a family of weak bisimulations between $\langle A, \alpha\rangle$ and $\langle B, \beta\rangle$.
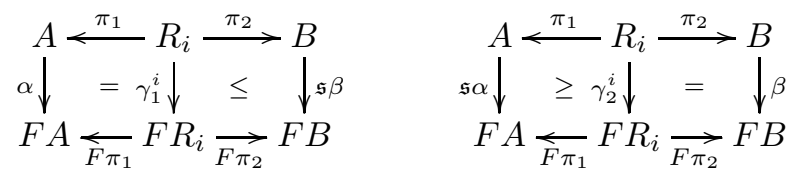

First consider the disjoint sum $\sum_{i \in I}\left\langle R_{i}, \gamma_{1}^{i}\right\rangle=\left\langle\sum_{i \in I} R_{i}, \gamma_{1}\right\rangle$. We will prove that given $\tau: \sum_{i \in I} R_{i} \rightarrow A \times B ;(r, i) \rightarrow r$ the mappings $p_{1}=\pi_{1} \circ \tau$ and $p_{2}=\pi_{2} \circ \tau$ satisfy:

$$
\begin{aligned}
& \alpha \circ p_{1}=F\left(p_{1}\right) \circ \gamma_{1}, \\
& \mathfrak{s} \beta \circ p_{2} \geq F\left(p_{2}\right) \circ \gamma_{1} .
\end{aligned}
$$


Note that for any $i \in I$ we have

$$
\alpha \circ p_{1} \circ e_{i}=\alpha \circ \pi_{1}=F\left(\pi_{1}\right) \circ \gamma_{1}^{i}=F\left(p_{1}\right) \circ F\left(e_{i}\right) \circ \gamma_{1}^{i}=F\left(p_{1}\right) \circ \gamma_{1} \circ e_{i} .
$$

Hence, $\alpha \circ p_{1}=F\left(p_{1}\right) \circ \gamma_{1}$. Moreover, for any $i \in I$ we have

$$
\mathfrak{s} \beta \circ p_{2} \circ e_{i}=\mathfrak{s} \beta \circ \pi_{2} \geq F\left(\pi_{2}\right) \circ \gamma_{1}^{i}=F\left(p_{2}\right) \circ F\left(e_{i}\right) \circ \gamma_{1}^{i}=F\left(p_{2}\right) \circ \gamma_{1} \circ e_{i} .
$$

By Lemma 1 it follows that $\mathfrak{s} \beta \circ p_{2} \geq F\left(p_{2}\right) \circ \gamma_{1}$.
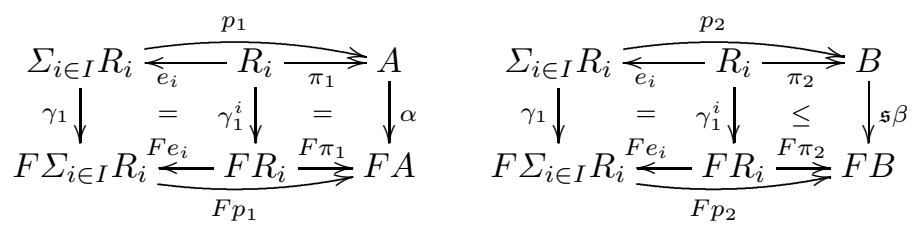

Note that the image of $\sum_{i \in I} R_{i}$ under the map $\tau: \sum_{i \in I} R_{i} \rightarrow A \times B$ is equal to $\bigcup_{i \in I} R_{i} \subseteq A \times B$. Put $\gamma_{1}^{\prime}: \bigcup_{i \in I} R_{i} \rightarrow F\left(\bigcup_{i \in I} R_{i}\right)$ so that, for any $r \in \bigcup_{i \in I} R_{i}$ there is $(r, i) \in \sum_{i \in I} R_{i}$ such that $\gamma_{1}^{\prime}(r)=F(\tau)\left(\gamma_{1}(r, i)\right)$. Observe that

$$
\begin{aligned}
& \alpha\left(\pi_{1}(r)\right)=\alpha\left(\pi_{1}(\tau(r, i))\right)=\alpha\left(p_{1}(r, i)\right)=F\left(p_{1}\right)\left(\gamma_{1}(r, i)\right)= \\
& =F\left(\pi_{1}\right)\left(F(\tau)\left(\gamma_{1}(r, i)\right)\right)=F\left(\pi_{1}\right) \circ \gamma_{1}^{\prime}(r), \\
& \mathfrak{s} \beta\left(\pi_{2}(r)\right)=\mathfrak{s} \beta\left(\pi_{2}(\tau(r, i))\right)=\mathfrak{s} \beta \circ p_{2}(r, i) \geq F\left(p_{2}\right) \circ \gamma_{1}(r, i)= \\
& =F\left(\pi_{2}\right) \circ F(\tau) \circ \gamma_{1}(r, i)=F\left(\pi_{2}\right) \circ \gamma_{1}^{\prime}(r) .
\end{aligned}
$$

Hence, $\alpha \circ \pi_{1}=F\left(\pi_{1}\right) \circ \gamma_{1}^{\prime}$ and $\mathfrak{s} \beta \circ \pi_{2} \geq F\left(\pi_{2}\right) \circ \gamma_{1}^{\prime}$. Similarily we prove existence of $\gamma_{2}^{\prime}: \bigcup_{i \in I} R_{i} \rightarrow F\left(\bigcup_{i \in I} R_{i}\right)$ possessing the desired properties and making $\bigcup_{i \in I} R_{i}$ a weak bisimulation.

The following lemma is an analogue of a similar result for standard bisimulations presented in e.g. [11] (Lemma 5.3). Moreover, the proof of Lemma 3 is a direct translation of the proof of the analogous result. Hence, we leave the following result without a proof.

Lemma 3. Let $X$ be a set and let $\xi_{1}: X \rightarrow F X$ and $\xi_{2}: X \rightarrow F X$ be two coalgebraic structures. Finally, let $f: X \rightarrow A, g: X \rightarrow B$ be mappings such that $f$ is a homomorphism from $\left\langle X, \xi_{1}\right\rangle$ to $\langle A, \alpha\rangle, g$ is a homomorphism from $\left\langle X, \xi_{2}\right\rangle$ to $\langle B, \beta\rangle$ and the mappings $f, g$ satisfy:
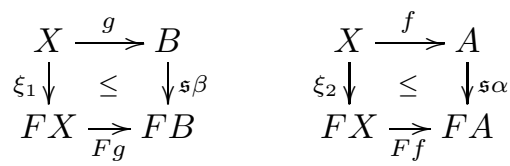

then the set $\langle f, g\rangle(X)=\{(f(x), g(x)) \in A \times B \mid x \in X\}$ is a weak bisimulation between $\langle A, \alpha\rangle$ and $\langle B, \beta\rangle$. 
Theorem 3. Let $F:$ Set $\rightarrow$ Set weakly preserve pullbacks and let $\langle A, \alpha\rangle,\langle B, \beta\rangle$ and $\langle C, \delta\rangle$ be $F$-coalgebras from the class $C$. Let $R_{1}$ be a weak bisimulation between $\langle A, \alpha\rangle$ and $\langle B, \beta\rangle$ and $R_{2}$ be a weak bisimulation between $\langle B, \beta\rangle$ and $\langle C, \delta\rangle$. Then

$$
R_{1} \circ R_{2}=\left\{(a, c) \mid \exists b \in B \text { s.t. }(a, b) \in R_{1} \text { and }(b, c) \in R_{2}\right\}
$$

is a weak bisimulation between $\langle A, \alpha\rangle$ and $\langle C, \delta\rangle$.

Corollary 1. If $F:$ Set $\rightarrow$ Set weakly preserves pullbacks then the greatest weak bisimulation on a coalgebra $\langle A, \alpha\rangle$ is an equivalence relation.

Definition 5. A relation $R \subseteq A \times B$ is said to be a saturated weak bisimulation between $\langle A, \alpha\rangle$ and $\langle B, \beta\rangle$ provided that there is a structure $\gamma: R \rightarrow F R$ for which the following diagram commutes:

$$
\begin{gathered}
A \stackrel{\pi_{1}}{\longleftarrow} R \stackrel{\pi_{2}}{\longrightarrow} B \\
\mathfrak{s} \alpha \downarrow=\gamma \downarrow=\downarrow_{\mathfrak{s} \beta}=\gamma \underset{F \pi_{1}}{\longrightarrow} F R \underset{F \pi_{2}}{\longrightarrow} B
\end{gathered}
$$

Remark 1. We see that a saturated weak bisimulation between $\langle A, \alpha\rangle$ and $\langle B, \beta\rangle$ is defined as a standard bisimulation between saturated models $\langle A, \mathfrak{s} \alpha\rangle$ and $\langle B, \mathfrak{s} \beta\rangle$. Hence, any property true for standard bisimulation is also true for a saturated weak bisimulation.

Proposition 2. Let $R \subseteq A \times B$ be a standard bisimulation between $\langle A, \alpha\rangle$ and $\langle B, \beta\rangle$. Then $R$ is also a saturated weak bisimulation between $\langle A, \alpha\rangle$ and $\langle B, \beta\rangle$.

Theorem 4. Let $F:$ Set $\rightarrow$ Set weakly preserve kernel pairs and let $R \subseteq A \times A$ be an equivalence relation which is a weak bisimulation on $\langle A, \alpha\rangle$. Then $R$ is a saturated weak bisimulation on $\langle A, \alpha\rangle$.

Proof. Let $\gamma_{1}: R \rightarrow F R$ be a structure for which $\alpha \circ \pi_{1}=F \pi_{1} \circ \gamma_{1}$ and $F \pi_{2} \circ \gamma_{1} \leq$ $\mathfrak{s} \alpha \circ \pi_{2}$. By properties of the saturator $\mathfrak{s}$ it follows that $\mathfrak{s} \alpha \circ \pi_{1}=F \pi_{1} \circ \mathfrak{s} \gamma_{1}$ and $F \pi_{2} \circ \mathfrak{s} \gamma_{1} \leq \mathfrak{s} \alpha \circ \pi_{2}$. In other words,

$$
\begin{gathered}
A \stackrel{\pi_{1}}{\stackrel{\pi_{2}}{\longrightarrow}} A \\
\mathfrak{s} \alpha \downarrow=\mathfrak{s} \Downarrow^{\leq} \leq \downarrow \mathfrak{s} \alpha \\
F A \underset{F \pi_{1}}{\stackrel{\leftarrow}{\leftrightarrows}} \underset{F \pi_{2}}{\longrightarrow} F A
\end{gathered}
$$

Let $p: A \rightarrow A / R ; a \mapsto a / R$. Since $F:$ Set $\rightarrow$ Set preserves kernel pairs the following diagram is a weak pullback diagram:

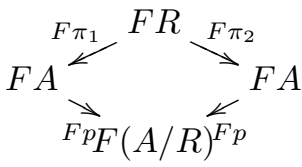


Since $F p \circ F \pi_{1}=F p \circ F \pi_{2}$ we have $F p \circ \mathfrak{s} \alpha \circ \pi_{1}=F p \circ F \pi_{1} \circ \mathfrak{s} \gamma_{1}=F p \circ F \pi_{2} \circ \mathfrak{s} \gamma_{1} \leq$ $F p \circ \mathfrak{s} \alpha \circ \pi_{2}$. Let $k: R \rightarrow R ;(a, b) \mapsto(b, a)$. We see that $F p \circ \mathfrak{s} \alpha \circ \pi_{1} \circ k \leq$ $F p \circ \mathfrak{s} \alpha \circ \pi_{2} \circ k$. Since $\pi_{1} \circ k=\pi_{2}$ and $\pi_{2} \circ k=\pi_{1}$ it follows that

$$
F p \circ \mathfrak{s} \alpha \circ \pi_{2} \leq F p \circ \mathfrak{s} \alpha \circ \pi_{1} .
$$

Hence, $F p \circ \mathfrak{s} \alpha \circ \pi_{1}=F p \circ \mathfrak{s} \alpha \circ \pi_{2}$. In other words, the set $R$ together with $\mathfrak{s} \alpha \circ \pi_{1}$ and $\mathfrak{s} \alpha \circ \pi_{2}$ is a cone over the diagram $F A \stackrel{F p}{\rightarrow} F(A / R) \stackrel{F p}{\leftarrow} F A$. Recall that $F R$ with $F \pi_{1}$ and $F \pi_{2}$ is a weak pullback of $F A \stackrel{F p}{\rightarrow} F(A / R) \stackrel{F p}{\leftarrow} F A$. The fact that $F$ preserves weak pullbacks provides us with a mediating morphism $\gamma: R \rightarrow F R$ satisfying

$$
\mathfrak{s} \alpha \circ \pi_{1}=F \pi_{1} \circ \gamma \text { and } \mathfrak{s} \alpha \circ \pi_{2}=F \pi_{2} \circ \gamma
$$

We say that two elements $a, b \in A$ are weakly bisimilar, and write $a \approx_{w} b$ if there is a weak bisimulation $R \subseteq A \times A$ on $\langle A, \alpha\rangle$ for which $(a, b) \in R$. We say that $a$ and $b$ are saturated weakly bisimilar, and write $a \approx_{s w} b$, if there is a saturated weak bisimulation $R$ on $\langle A, \alpha\rangle$ containing $(a, b)$.

Corollary 2. Let $F:$ Set $\rightarrow$ Set be a functor weakly preserving pullbacks. Then the relations $\approx_{w}$ and $\approx_{s w}$ are equivalence relations and

$$
\approx_{w} \subseteq \approx_{s w}
$$

Definition 6. We say that a functor $F$ : Set $\rightarrow$ Pos preserves downsets provided that for any $f: X \rightarrow Y$ and any $\boldsymbol{x} \in F X$ the following equality holds:

$$
F f(\boldsymbol{x} \downarrow)=F f\left(\left\{\boldsymbol{x}^{\prime} \in F X \mid \boldsymbol{x}^{\prime} \leq \boldsymbol{x}\right\}\right)=F f(\boldsymbol{x}) \downarrow=\{\boldsymbol{y} \in F Y \mid \boldsymbol{y} \leq F f(\boldsymbol{x})\} .
$$

It is easy to see that all functors from Example 2 preserve downsets. In the following example we will present a functor weakly preserving pullbacks and not preserving downsets for which the greatest weak bisimulation and the greatest saturated weak bisimulation do not always coincide.

Example 9. Define a functor $F:$ Set $\rightarrow$ Set by $F=\mathcal{I} d^{2}+\mathcal{I} d$. Clearly, the functor $F$ weakly preserves pullbacks. For any set $X$ let us introduce a partial order $\leq$ on $F X$ as the smallest partial order satisfying

$$
x \leq(x, x) \text { for any } x \in X
$$

The order $\leq$ is well defined and turns the functor $F$ into $F:$ Set $\rightarrow$ Pos. Now consider sets $X=\left\{x_{1}, x_{2}\right\}, Y=\{y\}$ and the unique mapping $f: X \rightarrow Y$. Take $(y, y) \in F Y$ and note that $y \leq(y, y)=\left(f\left(x_{1}\right), f\left(x_{2}\right)\right)=F f\left(\left(x_{1}, x_{2}\right)\right)$. In other words, $y \in F f\left(x_{1}, x_{2}\right) \downarrow$ and $\left(x_{1}, x_{2}\right) \downarrow=\left\{\left(x_{1}, x_{2}\right)\right\}$. Hence, the functor $F$ does not preserve downsets. For any $F$-coalgebra $\langle A, \alpha\rangle$ define an operator $\mathfrak{s} \alpha: A \rightarrow F A$ by

$$
\mathfrak{s} \alpha(a):=\text { if } \alpha(a)=b \text { then }(b, b) \text { else } \alpha(a) .
$$


The operator $\mathfrak{s}: \operatorname{Set}_{F} \rightarrow \operatorname{Set}_{F}$ is a coalgebraic saturator with respect to the class of all $F$-coalgebras. Now consider a set $A=\{x, y\}$ and define a structure $\alpha: A \rightarrow F A$ by $\alpha(x)=x$ and $\alpha(y)=(x, y)$. Clearly, $x \approx_{s w} y$ since $\mathfrak{s} \alpha(x)=(x, x)$ and $\mathfrak{s} \alpha(y)=(x, y)$ and if we put $R=\{(x, y),(x, x)\}$ then for $\gamma: R \rightarrow F R$ defined by $\gamma(x, y)=((x, x),(x, y)), \gamma(x, x)=((x, x),(x, x))$ we have $\mathfrak{s} \alpha \circ \pi_{1}=F \pi_{1} \circ \gamma$ and $\mathfrak{s} \alpha \circ \pi_{2}=F \pi_{2} \circ \gamma$. At the same time the states $x$ and $y$ are not weakly bisimilar. Indeed, if there was a weak bisimulation $R$ containing $(x, y)$ then it would imply existence of $\gamma_{1}: R \rightarrow F R$ satisfying $\alpha \circ \pi_{1}=F \pi_{1} \circ \gamma_{1}$ and $F \pi_{2} \circ \gamma_{1} \leq \mathfrak{s} \alpha \circ \pi_{2}$. Since $(x, y) \downarrow=\{(x, y)\}$ we would then have

$$
\begin{array}{r}
x=\alpha(x)=\alpha\left(\pi_{1}(x, y)\right)=F \pi_{1}(\gamma(x, y)), \\
(x, y)=\mathfrak{s} \alpha(y)=\mathfrak{s} \alpha\left(\pi_{2}(x, y)\right)=F \pi_{2}(\gamma(x, y))
\end{array}
$$

which is impossible.

Theorem 5. Let $F$ : Set $\rightarrow$ Set weakly preserve kernel pairs and preserve downsets. Let $R \subseteq A \times A$ be an equivalence relation which is a saturated weak bisimulation on $\langle A, \alpha\rangle$. Then $R$ is a weak bisimulation on $\langle A, \alpha\rangle$.

Proof. Let $\gamma: R \rightarrow F R$ be the structure for which $\mathfrak{s} \alpha \circ \pi_{1}=F \pi_{1} \circ \gamma$ and $F \pi_{2} \circ \gamma=\mathfrak{s} \alpha \circ \pi_{2}$. Let $p: A \rightarrow A / R ; a \mapsto a / R$. Since $F:$ Set $\rightarrow$ Set preserves kernel pairs the following diagram is a weak pullback diagram:

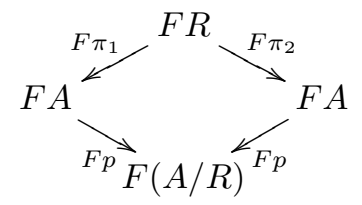

Consider the mappings $F p \circ \alpha \circ \pi_{1}$ and $F p \circ \mathfrak{s} \alpha \circ \pi_{2}$ and observe that

$$
\begin{aligned}
& F p \circ \alpha \circ \pi_{1} \leq F p \circ \mathfrak{s} \alpha \circ \pi_{1}= \\
& F p \circ F \pi_{1} \circ \gamma=F p \circ F \pi_{2} \circ \gamma=F p \circ \mathfrak{s} \alpha \circ \pi_{2} .
\end{aligned}
$$

This means that for any pair $(a, b) \in R$ we have $F p(\alpha(a)) \leq F p(\mathfrak{s} \alpha(b))$. In other words,

$$
F p(\alpha(a)) \in F p(\mathfrak{s} \alpha(b)) \downarrow
$$

Since $F$ preserves downsets, there exists an element $\boldsymbol{x} \in F A$ such that $\boldsymbol{x} \leq \mathfrak{s} \alpha(b)$ for which

$$
F p(\alpha(a))=F p(\boldsymbol{x}) .
$$

Because $F R$ together with $F \pi_{1}$ and $F \pi_{2}$ is a weak pullback of the diagram $F A \stackrel{F p}{\rightarrow} F(A / R) \stackrel{F p}{\leftarrow} F A$, there is an element $\boldsymbol{r}_{(a, b)} \in F R$ such that $F \pi_{1}\left(\boldsymbol{r}_{(a, b)}\right)=$ $\alpha(a)$ and $F \pi_{2}\left(\boldsymbol{r}_{(a, b)}\right)=\boldsymbol{x}$. Define $\gamma_{1}: R \rightarrow F R ;(a, b) \mapsto \boldsymbol{r}_{(a, b)}$. The structure $\gamma_{1}$ satisfies $\alpha \circ \pi_{1}=F \pi_{1} \circ \gamma_{1}$ and $F \pi_{2} \circ \gamma_{1} \leq \mathfrak{s} \alpha \circ \pi_{2}$. Similarily, we prove existence of $\gamma_{2}: R \rightarrow F R$ satisfying $\mathfrak{s} \alpha \circ \pi_{1} \geq F \pi_{1} \circ \gamma_{2}$ and $F \pi_{2} \circ \gamma_{2}=\alpha \circ \pi_{2}$.

Corollary 3. Let $S:$ Set $\rightarrow$ Set weakly preserve pullbacks and preserve downsets. Then for any $S$-coalgebra $\langle A, \alpha\rangle$ the relations $\approx_{w}$ and $\approx_{s w}$ are equivalence relations and

$$
\approx_{w}=\approx_{s w}
$$




\section{$5 \quad$ Weak Coinduction Principle}

In this section we assume that the saturator $\mathfrak{s}$ we work with is an operator defined on the whole category of $F$-coalgebras. In the category $\operatorname{Set}_{F}$ for any mapping $f: A \rightarrow B$ we have $f:\langle A, \alpha\rangle \rightarrow\langle B, \beta\rangle$ is a homomorphism if and only if the relation $\operatorname{gr}(f)=\{(a, f(a)) \in A \times B \mid a \in A\}$ is a standard bisimulation (see 2, 11]). This motivates considering the following category (F. Bonchi, personal communication). Let $\operatorname{Set}_{F}^{w}$ denote the category in which objects are standard $F$-coalgebras and in which a map $f: A \rightarrow B$ is a morphism between two objects $\langle A, \alpha\rangle$ and $\langle B, \beta\rangle$ provided that the relation $\operatorname{gr}(f)=\{(a, f(a)) \mid a \in A\}$ is a weak bisimulation.

Lemma 4. Let $\langle A, \alpha\rangle$ be an $F$-coalgebra and let a family $\left\langle S_{i}, \sigma_{i}\right\rangle$, where $S_{i} \subseteq A$, be a family of subcoalgebras of $\langle A, \alpha\rangle$ in $\operatorname{Set}_{F}$ such that $\mathfrak{s} \sigma_{i}=\sigma_{i}$. Then there is a structure $\sigma: \bigcup S_{i} \rightarrow F\left(\bigcup S_{i}\right)$ making $\left\langle\bigcup S_{i}, \sigma\right\rangle$ a subcoalgebra of $\langle A, \alpha\rangle$ and $\mathfrak{s} \sigma=\sigma$.

Corollary 4. Let $\langle A, \alpha\rangle$ be an F-coalgebra. There is the greatest subcoalgebra $\langle S, \sigma\rangle$ of $\langle A, \alpha\rangle$ such that $\mathfrak{s} \sigma=\sigma$.

Lemma 5. Let $\langle T, t\rangle$ be a terminal coalgebra in the category $\operatorname{Set}_{F}$. Then $\langle T, \mathfrak{s t}\rangle$ is a weakly terminal object in $\operatorname{Set}_{F}^{w}$.

Let $\left\langle T_{s}, t^{\prime}\right\rangle$ be the greatest subcoalgebra of the terminal coalgebra $\langle T, t\rangle$ in $\operatorname{Set}_{F}$ such that $\mathfrak{s} t^{\prime}=t^{\prime}$.

Lemma 6. If $F$ admits a terminal coalgebra $\langle T, t\rangle$ in $\operatorname{Set}_{F}$ and weakly preserves pullbacks then the greatest weak bisimulation on $\left\langle T_{s}, t^{\prime}\right\rangle$ is the equality relation.

Proof. Assume that for $x, y \in T_{s}$ we have $x \approx_{w} y$. This implies $x \approx_{s w} y$. Since, $\mathfrak{s} t^{\prime}=t^{\prime}$ this means that the saturated weak bisimulation $\approx_{s w}$ is a standard bisimulation on $\left\langle T_{s}, t^{\prime}\right\rangle$. Since, the coalgebra $\left\langle T_{s}, t^{\prime}\right\rangle$ is a subcoalgebra of the terminal coalgebra $\langle T, t\rangle$ the bisimulation $\approx_{s w}$ is an equality relation (see [211] for details). Hence $x=y$.

The above lemma allows us to formulate a weak coinduction principle for $\left\langle T_{s}, t^{\prime}\right\rangle$. For two states $x, y \in T_{s}$ we have $x \approx_{w} y \Longleftrightarrow x=y$.

Theorem 6. If $F$ admits a terminal coalgebra $\langle T, t\rangle$ in $\operatorname{Set}_{F}$ and weakly preserves pullbacks then the coalgebra $\left\langle T_{s}, t^{\prime}\right\rangle$ is a terminal object in $\operatorname{Set}_{F}^{w}$.

Proof. To prove that $\left\langle T_{s}, t^{\prime}\right\rangle$ is weakly terminal it is enough to construct a homomorphism in $\operatorname{Set}_{F}^{w}$ from $\langle T, \mathfrak{s t}\rangle$ to $\left\langle T_{s}, t^{\prime}\right\rangle$ and apply Lemma 5. Since $\langle T, t\rangle$ is terminal in $\operatorname{Set}_{F}$ there exists a unique homomorphism $\llbracket-\rrbracket_{\mathfrak{s} t}:\langle T, \mathfrak{s t}\rangle \rightarrow\langle T, t\rangle$. Any homomorphic image is a subcoalgebra of the codomain (see [2, [1] for details). Therefore, we can consider $\llbracket-\rrbracket_{\mathfrak{s} t}$ as an onto homomorphism between $\langle T, \mathfrak{s t}\rangle$ and $\left\langle\llbracket T \rrbracket_{\mathfrak{s} t}, t_{\left|\llbracket T \rrbracket_{\mathfrak{s} t}\right\rangle}\right\rangle$, where the object $\left\langle\llbracket T \rrbracket_{\mathfrak{s} t}, t_{\mid \llbracket T \rrbracket_{\mathfrak{s} t}}\right\rangle$ is a subcoalgebra of $\langle T, t\rangle$ in $\operatorname{Set}_{F}$. In other words, we have 


$$
\begin{aligned}
& \begin{array}{c}
T \stackrel{\llbracket-\rrbracket_{\mathfrak{s} t}}{\longrightarrow} \llbracket T \rrbracket_{\mathfrak{s} t} \\
\mathfrak{s t} \downarrow=\quad \downarrow^{t_{\mid \llbracket T \rrbracket_{\mathfrak{s} t}}}
\end{array} \\
& F T \underset{F \llbracket-\rrbracket_{\mathfrak{s} t}}{\longrightarrow}\left[T \rrbracket \rrbracket_{\mathfrak{s} t}\right.
\end{aligned}
$$

Hence,

$$
\begin{aligned}
& T \stackrel{\llbracket-\rrbracket_{\mathfrak{s} t}}{\longrightarrow} \llbracket T \rrbracket_{\mathfrak{s} t} \\
& \mathfrak{s t} \downarrow=\downarrow^{\mathfrak{s} t \mid \llbracket T \rrbracket_{\mathfrak{s} t}} \\
& F T \underset{F \llbracket-\rrbracket_{\mathfrak{s} t}}{\longrightarrow} \llbracket T \rrbracket_{\mathfrak{s} t}
\end{aligned}
$$

Therefore, $\mathfrak{s t}_{\mid \llbracket T \rrbracket_{\mathfrak{s} t}}=t_{\mid \llbracket T \rrbracket_{\mathfrak{s} t}}$ and $\left\langle\llbracket T \rrbracket_{\mathfrak{s} t}, t_{\mid \llbracket T \rrbracket_{\mathfrak{s} t}}\right\rangle$ is a subcoalgebra of $\left\langle T_{s}, t^{\prime}\right\rangle$. For uniqueness consider two homomorphisms $f_{1}, f_{2}$ from an $F$-coalgebra $\langle A, \alpha\rangle$ to $\left\langle T_{s}, t^{\prime}\right\rangle$ in $\operatorname{Set}_{F}^{w}$. This means that the relations $\operatorname{gr}\left(f_{1}\right)$ and $\operatorname{gr}\left(f_{2}\right)$ are weak bisimulations between $\langle A, \alpha\rangle$ and $\left\langle T_{s}, t^{\prime}\right\rangle$. By the properties of weak bisimulations the relation $\operatorname{gr}\left(f_{1}\right)^{-1} \circ \operatorname{gr}\left(f_{2}\right)=\left\{\left(f_{1}(a), f_{2}(a)\right) \mid a \in A\right\}$ is a weak bisimulation on $\left\langle T_{s}, t^{\prime}\right\rangle$. By Lemma 6 we get that $f_{1}(a)=f_{2}(a)$ for any $a \in A$.

For an $F$-coalgebra $\langle A, \alpha\rangle$ let $\llbracket-\rrbracket_{\alpha}^{w}$ denote the unique homomorphism from $\langle A, \alpha\rangle$ to $\left\langle T_{s}, t^{\prime}\right\rangle$ in $\operatorname{Set}_{F}^{w}$. We see in the proof of Theorem 6 that $\llbracket-\rrbracket_{\alpha}^{w}=$ $\llbracket-\rrbracket_{\mathfrak{s} t} \circ \llbracket-\rrbracket_{\alpha}$.

Theorem 7. Let $F$ weakly preserve pullbacks. For two elements $a, b \in A$ we have

$$
a \approx_{w} b \Longleftrightarrow \llbracket a \rrbracket_{\alpha}^{w}=\llbracket b \rrbracket_{\alpha}^{w}
$$

Proof. Assume $a \approx_{w} b$. Since $\llbracket-\rrbracket_{\alpha}^{w}$ is a homomorphism in $\operatorname{Set}_{F}^{w}$ the relation $\operatorname{gr}\left(\llbracket-\rrbracket_{\alpha}^{w}\right)$ is a weak bisimulation between $\langle A, \alpha\rangle$ and $\left\langle T_{s}, t^{\prime}\right\rangle$. Since $F$ weakly preserves pullbacks the relation $g r\left(\llbracket-\rrbracket_{\alpha}^{w}\right)^{-1} \circ \approx_{w} \circ g r\left(\llbracket-\rrbracket_{\alpha}^{w}\right)$ is a weak bisimulation on $\left\langle T_{s}, t^{\prime}\right\rangle$ such that

$$
\left(\llbracket a \rrbracket_{\alpha}^{w}, \llbracket b \rrbracket_{\alpha}^{w}\right) \in \operatorname{gr}\left(\llbracket-\rrbracket_{\alpha}^{w}\right)^{-1} \circ \approx_{w} \circ g r\left(\llbracket-\rrbracket_{\alpha}^{w}\right) .
$$

By Lemma 6 we get that $\llbracket a \rrbracket_{\alpha}^{w}=\llbracket b \rrbracket_{\alpha}^{w}$. Conversely, let $\llbracket a \rrbracket_{\alpha}^{w}=\llbracket b \rrbracket_{\alpha}^{w}$. This means that the weak bisimulation $g r\left(\llbracket-\rrbracket_{\alpha}^{w}\right) \circ g r\left(\llbracket-\rrbracket_{\alpha}^{w}\right)^{-1}$ on $\langle A, \alpha\rangle$ contains a pair $(a, b)$. Hence, $a \approx_{w} b$.

\section{Summary and Future Work}

In this paper we introduced a coalgebraic setting in which we can define weak bisimulation in two ways generalizing Definition 1 and Definition 2 and compared them. We showed that the definitions coincide with the standard definitions of weak bisimulation for labelled transition systems and simple Segala systems. The approach towards defining weak bisimulation presented in this paper has two main advantages. First of all, it is a very general and simple approach. In 
particular it does not require an explicit specification of the observable and unobservable part of the functor. Second of all, it easily captures the computational aspects of weak bisimilarity. It is worth noting that it has some limitations. Part of the author's ongoing research is to establish the reason why it does not work for e.g. fully probabilistic processes introduced in [1] and studied from the perspective of coalgebra in [9]. Moreover, it may seem that the setting presented in the paper is too general. To justify the statement note that for instance for an LTS coalgebra $\langle A, \alpha\rangle$ we may define a saturator as follows:

$$
\mathfrak{s} \alpha(a):=\{(\tau, a)\} \cup\left\{\left(\sigma, a^{\prime}\right) \mid a \stackrel{\tau^{*}}{\rightarrow} \circ \stackrel{\sigma}{\rightarrow} a^{\prime}\right\} .
$$

The above definition of a saturator would lead to a different definition of weak bisimulation for LTS. Therefore, it is necessary to establish more concrete ways for definining standard saturators of coalgebras that lead to standard definitions of weak bisimulations.

Acknowledgements. I would like to thank Jan Rutten for many interesting comments and hospitality in CWI, Amsterdam, where part of the work on this paper was completed. I am very grateful to F. Bonchi, M. Bonsangue, H. Hansen, B. Klin and A. Silva for various suggestions for improvement of my results and for future work. I would also like to express my gratitude to anonymous referees of the paper for valuable comments and remarks.

\section{References}

1. Baier, C., Hermanns, H.: Weak Bisimulation for Fully Probabilistic Processes. In: Grumberg, O. (ed.) CAV 1997. LNCS, vol. 1254, pp. 119-130. Springer, Heidelberg (1997)

2. Gumm, H.P.: Elements of the general theory of coalgebras. In: LUATCS 1999, Rand Afrikaans University, Johannesburg (1999)

3. Hughes, J., Jacobs, B.: Simulations in coalgebra. TCS 327(1-2), 71-108 (2004)

4. Milner, R.: A Calculus of Communication Systems. LNCS, vol. 92. Springer, Heidelberg (1980)

5. Rothe, J.: A syntactical approach to weak (bi)-simulation for coalgebras. In: Moss, L. (ed.) Proc. CMCS 2002. ENTCS, vol. 65, pp. 270-285 (2002)

6. Rothe, J., Masulović, D.: Towards weak bisimulation for coalgebras. In: Proc. Categorical Methods for Concurrency, Interaction and Mobility. ENTCS, vol. 68(1), pp. 32-46 (2002)

7. Segala, R., Lynch, N.: Probabilistic Simulations for Probabilistic Processes. In: Jonsson, B., Parrow, J. (eds.) CONCUR 1994. LNCS, vol. 836, pp. 481-496. Springer, Heidelberg (1994)

8. Segala, R.: Modeling and verification of randomized distributed real-time systems. Ph.D. thesis. MIT (1995)

9. Sokolova, A., de Vink, E., Woracek, H.: Coalgebraic Weak Bisimulation for ActionType Systems. Sci. Ann. Comp. Sci. 19, 93-144 (2009)

10. Rutten, J.J.M.M.: A note on coinduction and weak bisimilarity for while programs. Theoretical Informatics and Applications (RAIRO) 33, 393-400 (1999)

11. Rutten, J.J.M.M.: Universal coalgebra: a theory of systems. TCS 249(1), 3-80 (2000) 


\section{Appendix}

Proof (Lemma 2). Let $\mathfrak{s}$ be a saturator and let $\langle A, \alpha\rangle \in \mathrm{C}$. We see that for the identity mapping $i d_{A}: A \rightarrow A$ we have

$$
F\left(i d_{A}\right) \circ \mathfrak{s} \alpha=\mathfrak{s} \alpha \leq \mathfrak{s} \alpha=\mathfrak{s} \alpha \circ i d_{A}
$$

This implies that $F\left(i d_{A}\right) \circ \alpha \leq \mathfrak{s} \alpha \circ i d_{A}$. Hence, $\alpha \leq \mathfrak{s} \alpha$. We see that by extensivity rule and the assumption $\mathfrak{s}(\mathrm{C}) \subseteq \mathrm{C}$ we get $\mathfrak{s} \alpha \leq \mathfrak{s}(\mathfrak{s} \alpha)$. Therefore, to prove idempotency it is enough to show that $\mathfrak{s}(\mathfrak{s} \alpha) \leq \mathfrak{s} \alpha$. To do this consider the following inequality $F\left(i d_{A}\right) \circ \mathfrak{s} \alpha=\mathfrak{s} \alpha \leq \mathfrak{s} \alpha=\mathfrak{s} \alpha \circ i d_{A}$. By order preservation it implies that $F\left(i d_{A}\right) \circ \mathfrak{s}(\mathfrak{s} \alpha) \leq \mathfrak{s} \alpha \circ i d_{A}$. Thus, $\mathfrak{s}(\mathfrak{s} \alpha) \leq \mathfrak{s} \alpha$. Finally, consider two coalgebras $\langle A, \alpha\rangle$ and $\langle B, \beta\rangle$ from $C$ and a mapping $f: A \rightarrow B$ such that $F f \circ \alpha \leq \beta \circ f$. Note that $F f \circ \alpha \leq \beta \circ f \leq \mathfrak{s} \beta \circ f$. Since $F f \circ \alpha \leq \mathfrak{s} \beta \circ f$ then by the fact that $\mathfrak{s}$ is a saturator it follows that $F f \circ \mathfrak{s} \alpha \leq \mathfrak{s} \beta \circ f$.

Now in order to prove the converse consider a coalgebraic operator $\mathfrak{s}: \mathrm{C} \rightarrow \mathrm{C}$ which is a closure operator (i.e. is extensive, idempotent and monotonic). Let $\langle A, \alpha\rangle$ and $\langle B, \beta\rangle$ be coalgebras from $C$ and let $f: A \rightarrow B$ be a mapping. Assume that $F f \circ \alpha \leq \mathfrak{s} \beta \circ f$. By idempotency and monotonicity we get $F f \circ \mathfrak{s} \alpha \leq \mathfrak{s} \beta \circ f$. By extensivity we conclude that $F f \circ \alpha \leq \mathfrak{s} \beta \circ f$.

Proof (Lemma 4). Let $\sigma: \bigcup S_{i} \rightarrow F\left(\bigcup S_{i}\right)$ be the unique structure making $\left\langle\bigcup S_{i}, \sigma\right\rangle$ a subcoalgebra of $\langle A, \alpha\rangle$ (such a structure always exists [2, 10]). Let $e_{i}: S_{i} \rightarrow \bigcup S_{i}$ denote the inclusions. Then $\sigma \circ e_{i}=F\left(e_{i}\right) \circ \sigma_{i}$. Since $\mathfrak{s}$ is a saturator this means that $\mathfrak{s} \sigma \circ e_{i}=F\left(e_{i}\right) \circ \mathfrak{s} \sigma_{i}=F\left(e_{i}\right) \circ \sigma_{i}$. Therefore, for any $i$ we have $\mathfrak{s} \sigma \circ e_{i}=\sigma \circ e_{i}$. Hence, $\mathfrak{s} \sigma=\sigma$.

Proof (Lemma 5). Let $\langle A, \alpha\rangle$ be any $F$-coalgebra. Let $\llbracket-\rrbracket_{\alpha}:\langle A, \alpha\rangle \rightarrow\langle T, t\rangle$ be the unique homomorphism in $\operatorname{Set}_{F}$ with $\langle T, t\rangle$ as codomain. Then $\llbracket-\rrbracket_{\alpha}$ is also a homomorphism between $\langle A, \mathfrak{s} \alpha\rangle$ and $\langle T, \mathfrak{s t}\rangle$. Since $\llbracket-\rrbracket_{\alpha}$ is a standard homomorphim the relation $\operatorname{gr}\left(\llbracket-\rrbracket_{\alpha}\right)=\left\{\left(a, \llbracket a \rrbracket_{\alpha}\right) \mid a \in A\right\}$ is a standard bisimulation between $\langle A, \alpha\rangle$ and $\langle T, t\rangle$. I.e. there is $\gamma: \operatorname{gr}\left(\llbracket-\rrbracket_{\alpha}\right) \rightarrow F\left(\operatorname{gr}\left(\llbracket-\rrbracket_{\alpha}\right)\right)$ such that

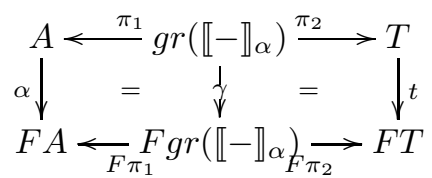

Since $t \leq \mathfrak{s t}$ and $\mathfrak{s s t}=\mathfrak{s t}$ this implies that

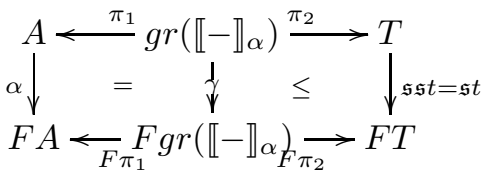


Moreover, by saturating the same diagram we get

$$
\begin{aligned}
& \begin{array}{c}
A \longleftarrow \\
\mathfrak{s} \alpha \downarrow=
\end{array}
\end{aligned}
$$

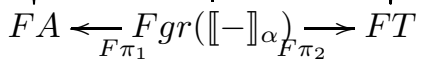

This means that $g r\left(\llbracket-\rrbracket_{\alpha}\right)$ together with $\gamma$ and $\mathfrak{s} \gamma$ is a weak bisimulation between $\langle A, \alpha\rangle$ and $\langle T, \mathfrak{s t}\rangle$ which concludes the proof. 